\title{
The Feasibility and Efficacy of Laparoscopic Extended Total Mesorectal Excision for Locally Advanced Lower Rectal Cancer
}

\author{
TAKASHI NONAKA, AKIKO FUKUDA, KYOICHIRO MAEKAWA, \\ SHIGEKI NAGAYOSHI, TAKAYUKI TOKUNAGA, MITSUTOSHI TAKATSUKI, \\ TOMOO KITAJIMA, KEN TANIGUCHI and HIKARU FUJIOKA
}

Department of Surgery, National Hospital Organization Nagasaki Medical Center, Nagasaki, Japan

\begin{abstract}
Background/Aim: Extended total mesorectal excision (ETME) is defined as en bloc resection of the adjacent organs outside the mesorectal fascia, that is indicated in cases with locally advanced lower rectal cancer (T4 tumor). The aim of this study was to evaluate the clinical and oncological outcomes of laparoscopic ETME (L-ETME) for locally advanced lower rectal cancer. Patients and Methods: The present study analyzed clinical outcomes and oncological outcomes of 11 consecutive patients who underwent L-ETME for cT4 lower rectal cancer in Nagasaki Medical Center between 2012 and 2015. Results: Of the 11 patients, 7 underwent neoadjuvant therapy, and 7 underwent pelvic node dissection. One case (7.1\%) underwent resection of anterior organs (prostate), 6 cases (54.5\%) had resection of the lateral organs (neurovascular bundle, hypogastric nerve, pelvic plexus, ovary, and internal iliac blood vessels) and 4 cases $(36.4 \%)$ had resection of both anterior and lateral organs. In all cases enrolled in this study, RO resection was achieved. The median operation time and intraoperative blood loss were $416 \mathrm{~min}$ and $350 \mathrm{ml}$, respectively. The postoperative complication rate was $18.2 \%$ (2/11). The 3-year overall survival rate was $79.5 \%$, and the 3-year local recurrence-free survival rate was $87.5 \%$. There was no mortality and no re-operation in this series. Conclusion: The results of the present study suggest that $L$ ETME is feasible and has efficacy for locally advanced lower rectal cancer.
\end{abstract}

This article is freely accessible online.

Correspondence to: Takashi Nonaka, Department of Surgery, National Hospital Organization Nagasaki Medical Center, 2-10011 Kubara, Ohmura, Nagasaki 856-8562, Japan. Tel: +81 957523121, Fax: +81 957540292, e-mail: tnonaka@ nagasaki-u.ac.jp

Key Words: Locally advanced lower rectal cancer, laparoscopic surgery, extended TME.
With improvements in surgical techniques, instruments, and experience, laparoscopic surgery (LS) has been successfully adopted for colorectal surgery because of its favorable effects since Jacob's first report in 1991 (1-5). Laparoscopic approaches offer decreased surgical trauma, fewer perioperative complications, and faster postoperative recovery with similar survival rates compared to open surgery (1-5). Recently, several experienced colorectal surgeons attempted LS even for locally advanced rectal cancers (T4 tumors). However, there's still skepticism on this use of LS, and many surgeons still consider it a relative contraindication because LS especially for advanced lower rectal cancer is a technically demanding approach in the confined narrow bony pelvis and has been associated with increased operation time, blood loss, and higher risks of conversion to open surgery or positive resection margins $(6,7)$. Moreover, the long-term oncologic outcomes after LS for T4-rectal cancer remain unclear due to the lack of high-quality, randomized, controlled trials (8-11). On the other hand, it has been reported that multi-visceral resection for T4-colorectal cancer has acceptable morbidity and mortality rates and a fair long-term prognosis $(5-7,12)$. In addition, several reports suggest that minimally invasive surgery such as a laparoscopic approach for multi-visceral resection could help achieve adequate short-term outcomes and improvements in quality of life $(5,13-15)$. However, whether the laparoscopic approach is safe and effective for locally advanced lower rectal cancer remains controversial.

We consider that the high-definition, illuminated, and magnified images obtained by laparoscopy provide a safer and more precise surgery for lower rectal cancer in the narrow pelvis $(6,7)$, even in extended surgery for locally advanced lower rectal cancer. We have been aggressively performing LS including laparoscopic total mesorectal excision (L-TME) and en bloc resection of the adjacent organs outside the plane of total mesorectal fascia to extend TME (L-ETME).

Our experience with L-ETME for advanced lower rectal cancer (clinical T4 tumors) is reported, and the feasibility and efficacy of this procedure were verified. 

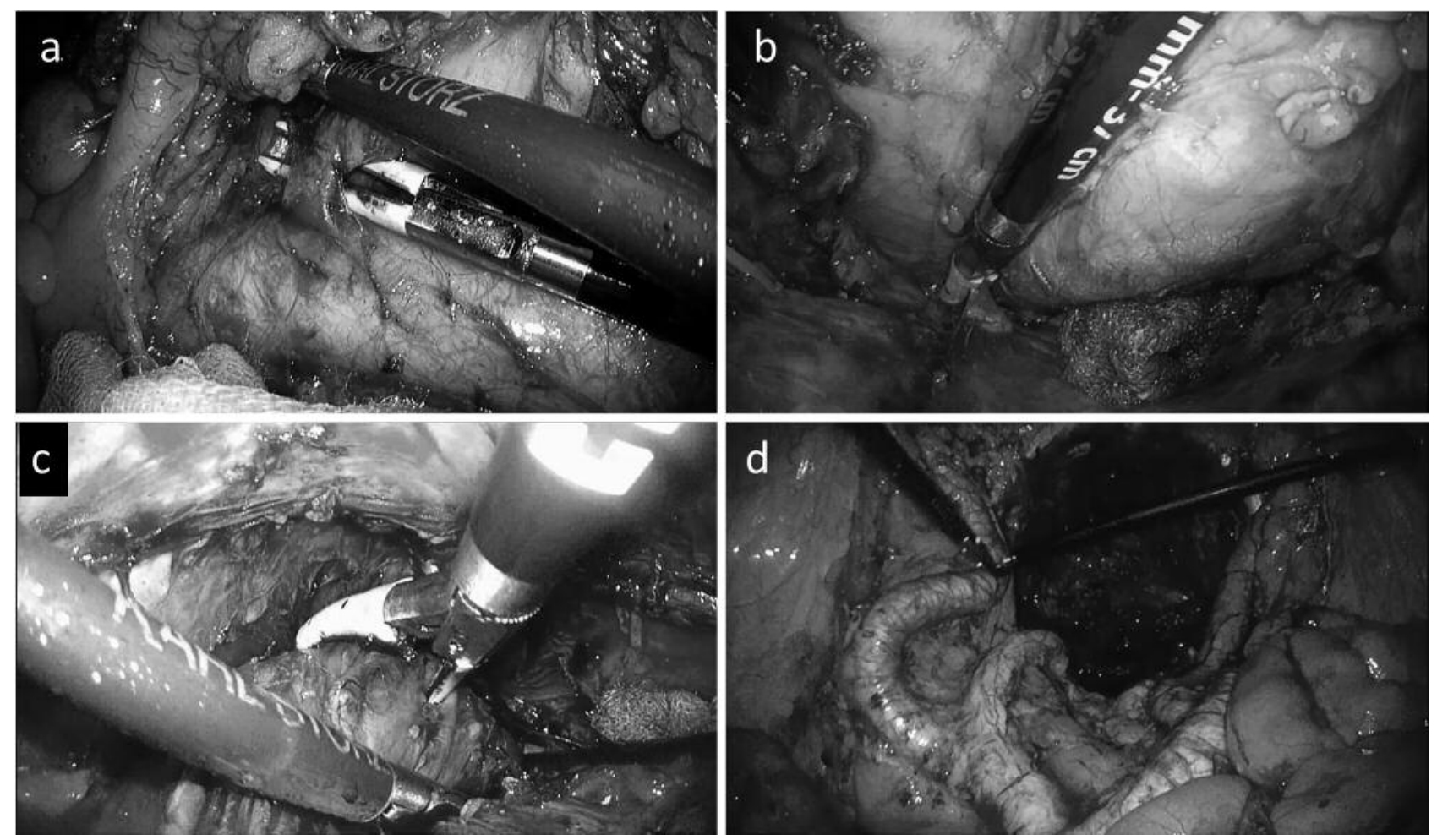

Figure 1. Operative technique. a) Dissection of the root of the inferior mesenteric artery; b) dissection of the posterior rectal space; $c$ ) divided DVC by Ligasure ${ }^{T M}$ Maryland (Covidien); d) operative view after total pelvic exenteration with en bloc lateral pelvic lymph node dissection.

\section{Materials and Methods}

A total of 11 patients with locally advanced lower rectal cancer underwent L-ETME in Nagasaki Medical Center between January 2012 and December 2015. All of these patients had advanced lower rectal cancer with infiltration into the outside mesorectum or with a high risk of a positive circumferential resection margin (CRM). In the cases with tumor invasion to the anterior side, multi-visceral resection of seminal vesicles, prostate, uterus, and/or vagina resection was performed, whereas in those with invasion to the lateral side, resections of the pelvic plexus, hypogastric nerve, internal iliac blood vessels, and/or neurovascular bundle were done. In all cases, the operative dissection line was determined on preoperative magnetic resonance imaging (MRI) images. Lateral pelvic node (LPN) dissection was indicated when LPN metastasis was suspected on the preoperative MRI images. In patients with suspected deep tumor infiltration and/or positive pelvic node involvement, neoadjuvant chemotherapy (NAC) or neoadjuvant chemoradiotherapy (NACRT) was performed based on the decision of a multi-disciplinary treatment team conference. L-ETME was performed by a team led by a specialist in colorectal surgery and endoscopic surgery certified by the Japan Society of Endoscopic Surgery. Medical records of all patients enrolled in this study were reviewed retrospectively, including patient characteristics, surgical outcomes, postoperative complications, and oncological outcomes. Postoperative complications were defined according to the Clavien-
Dindo classification (16). Postoperative death within 90 days after operation was defined as operative mortality. Overall survival probability was estimated with the Kaplan-Meier method. This study was approved by the Nagasaki Medical Center Ethics Committee.

Operative Technique: Laparoscopic total pelvic exenteration (Figure 1). Laparoscopic total pelvic exenteration as L-ETME for cases with anterior organ infiltration is presented. After the pneumoperitoneum, five ports were placed as follows: $12-\mathrm{mm}$ ports at the umbilicus and the lower right quadrant and 5-mm ports at the upper right, left, and lower left quadrants. First, the left side of the colon was mobilized using a medial-lateral retroperitoneal approach, and lymphadenectomy around the abdominal aorta and ligation of the inferior mesenteric artery were performed. Next, the posterior rectal space and lateral space were dissected. The dissection of lateral side lymph nodes was performed along the external iliac artery with preservation of the obturator nerve. Branches of the internal iliac vessels were each clipped and divided, and en bloc lateral lymph node dissection was performed simultaneously. After finishing the dissection of the posterior and lateral side lymph nodes, an additional 5-mm port was placed in the midline of the lower abdomen, and the dissection of the front lymph nodes was started. The space of Retzius was dissected, and the dorsal vein complex (DVC) was exposed. After bunching ligation of the DVC, the DVC was carefully dissected with Ligasure ${ }^{\mathrm{TM}}$ Maryland 
Table I. Patient characteristics.

\begin{tabular}{lc}
\hline Parameters & \\
\hline Age (years) & 65.0 \\
Gender (M/F) & $7 / 4$ \\
cT4a / cT4b & $8 / 3$ \\
Neoadjuvant therapy & $7(63.6 \%)$ \\
\hline
\end{tabular}

$(n=11)$

Table II. Multi-visceral resection.

\begin{tabular}{|c|c|c|}
\hline \multicolumn{3}{|c|}{ Resected organ } \\
\hline \multicolumn{2}{|c|}{ Anterior side } & $1(9.1 \%)$ \\
\hline \multicolumn{2}{|c|}{ Prostate (shaved) } & 1 \\
\hline \multicolumn{2}{|c|}{ Lateral side } & $6(54.5 \%)$ \\
\hline \multicolumn{2}{|c|}{ Neurovascular bundle } & 1 \\
\hline \multicolumn{2}{|c|}{ Pelvic plexus } & 3 \\
\hline \multicolumn{2}{|c|}{ Pelvic plexus + internal iliac vessels } & 1 \\
\hline \multicolumn{2}{|c|}{ Pelvic plexus + ovary } & 1 \\
\hline Anterior side & $+\quad$ Lateral side & $4(36.4 \%)$ \\
\hline Vagina (wall) & $\begin{array}{c}\text { Pelvic plexus }+ \\
\text { internal iliac vessels }\end{array}$ & 1 \\
\hline $\begin{array}{c}\text { Bladder }+ \text { vagina }+ \\
\text { uterus }\end{array}$ & $\begin{array}{c}\text { Pelvic plexus }+ \\
\text { internal iliac vessels }\end{array}$ & 1 \\
\hline Seminal vesicle & $\begin{array}{c}\text { Pelvic plexus }+ \\
\text { internal iliac vessels }\end{array}$ & 1 \\
\hline $\begin{array}{l}\text { Seminal Vesicle }+ \\
\text { prostate (shaved) }\end{array}$ & Pelvic plexus & 1 \\
\hline
\end{tabular}

(Medtronic, Mineapolis, MN, USA), and the urethra was clipped and dissected. Finally, the resected specimen was removed from the perineal wound. An ileal conduit and a sigmoidostomy were then constructed extracorporeally with a 5-cm laparotomy in the lower abdominal midline.

\section{Results}

Patient characteristics. The medical records of 11 patients with R0 resection were reviewed (Table I). Neoadjuvant therapy (NACRT: $n=3$ or NAC: $n=4$ ) was performed in 7 cases $(63.6 \%)$.

Operative results. Operative results are shown in Tables II and III. There were no conversions to open surgery. LPN dissection was performed in 7 cases $(63.6 \%)$, whereas multivisceral resection was performed in 4 cases $(36.4 \%)$. There were 6 patients (54.5\%) with lateral organ resection (including neurovascular bundle, hypogastric nerve, pelvic plexus, internal iliac blood vessels, and ovary), 1 with anterior organ resection (prostate resection), and 4 (36.4\%) with organ resections in the anterior and lateral sides (Table II). The

Table III. Operative results.

Parameters

Operation time (min)

Operative blood loss (ml)

Pelvic lymphonode dissection

Multi-visceral resection

Postoperative complication (G2/G3/G4)
416 (range $=257-714 \mathrm{~min})$

$350($ range $=20-1055 \mathrm{ml})$

$7(63.6 \%)$

$4(36.4 \%)$

$1 / 1 / 0$

$(\mathrm{n}=11)$
Table IV. Pathological findings.

\begin{tabular}{lc}
\hline Parameters & \\
\hline $\mathrm{pT} 4 \mathrm{a} / \mathrm{pT} 4 \mathrm{~b}$ & $8 / 3$ \\
$\mathrm{pN}(0 / 1 / 2 / 3)$ & $5 / 2 / 2 / 2$ \\
$\mathrm{pM}(0 / 1)$ & $8 / 3$ \\
$\mathrm{pStage}(\mathrm{II} / \mathrm{III} / \mathrm{IV})$ & $5 / 4 / 3$ \\
R0/R1 & $11 / 0$ \\
\hline & $(\mathrm{n}=11)$
\end{tabular}

median operation time was 416 min (257-714), and median intraoperative blood loss was $350 \mathrm{ml}(20-1055 \mathrm{ml})$. Only two postoperative complications (18.2\%) occurred, which were Grade 2 and Grade 3 each according to the Clavien-Dindo classification (e.g. anastomotic leakage and neurogenic bladder) (Table III). There was no operative mortality.

Pathological findings. Postoperative pathological findings according to the pTNM classification were pT4a $(8 / 11$, $72.7 \%)$ and pT4b (3/11, 27.3\%). In the pStage IV cases, 2 cases had liver metastases, and 1 case had metastasis to the para-aortic lymph node (Table IV).

Oncological outcomes. Short-term follow-up was carried out. The median follow-up was 19.5 months (range $=4-48$ months). The 3-year overall survival (OS) rate was 79.5\%, and the 3-year local recurrence-free survival (LRFS) rate was $87.5 \%$ (Figures 2 and 3 ).

\section{Discussion}

Complete surgical resection is important to achieve curative treatment for malignancy. Especially in locally advanced lower rectal cancer, TME and/or ETME with multi-visceral resection is often demanded to ensure a negative circumferential resection margin and to avoid perforation of tumor cells $(7,17$, 18). Currently, the indications for laparoscopic surgery for colorectal cancers have increased dramatically. However, the feasibility, applicability, and safety of L-TME or L-ETME with multi-visceral resection for advanced rectal cancer have not yet 


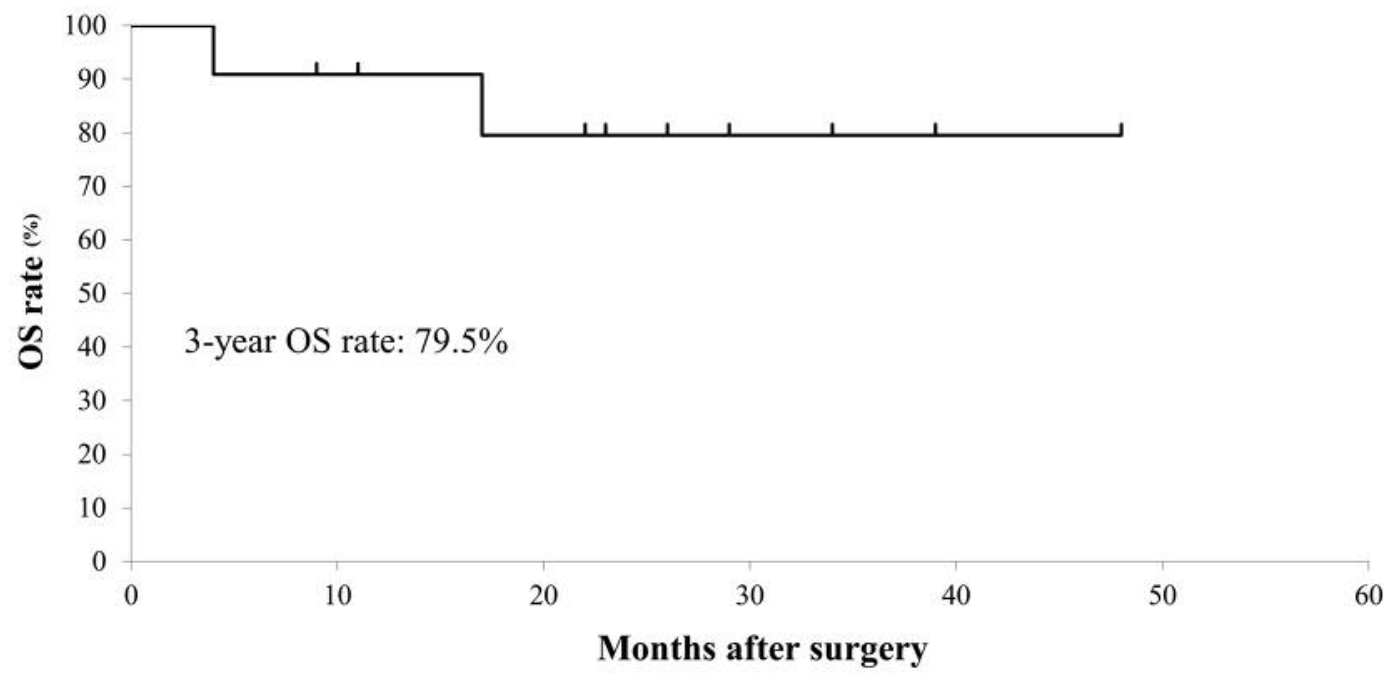

Figure 2. Overall survival (OS) $(n=11)$.

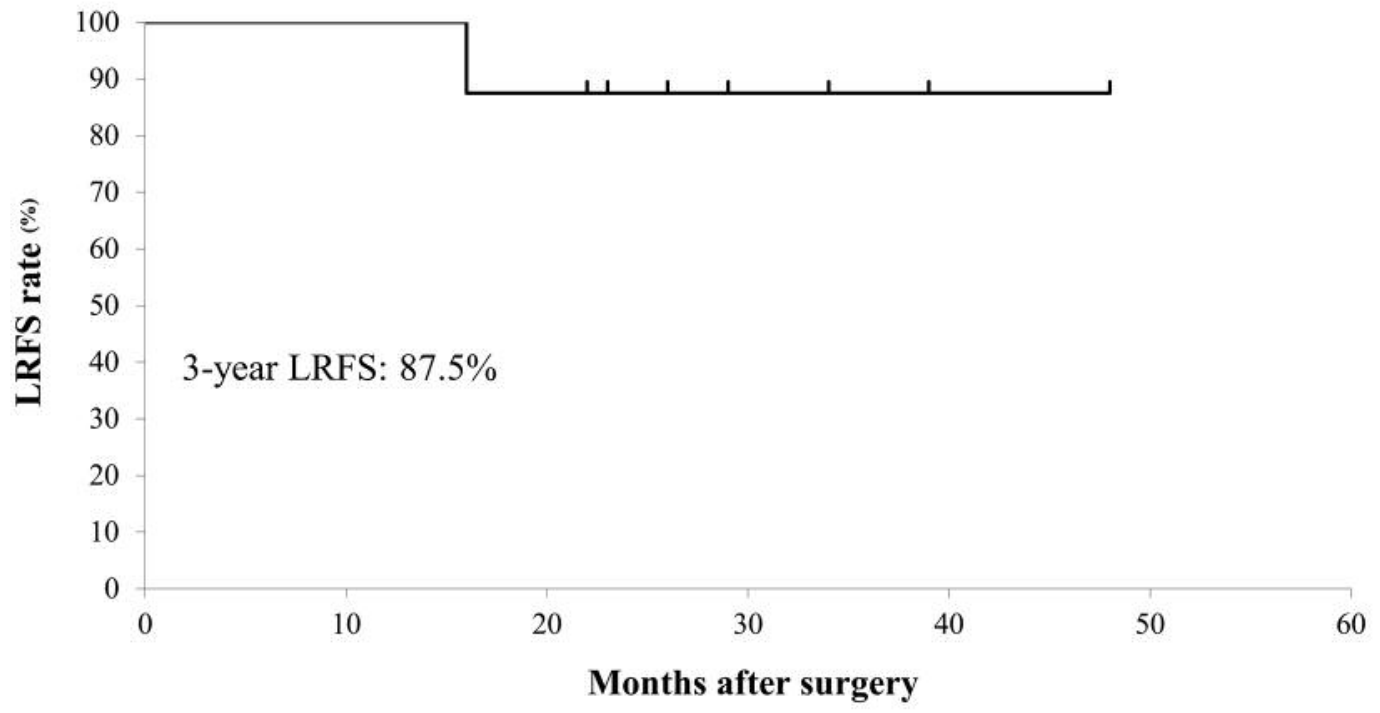

Figure 3. Local recurrence-free survival (LRFS) $(n=11)$.

been determined. Many colorectal surgeons still consider these procedures to be associated with technical difficulties, more blood loss, and longer operation time because of the complicated anatomy and narrow surgical field in the pelvis. Moreover, current high-quality, randomized, controlled trials, including the Medical Research Council Conventional vs. Laparoscopic Surgery in Colorectal Cancer (MRC CLASICC), Comparison of Open versus Laparoscopic Surgery for Mid or Low Rectal Cancer After Neoadjuvant Chemoradiotherapy (COREAN), and Colorectal Cancer Laparoscopic or Open
Resection (COLOR) II trials, excluded cases with T4 rectal cancer (6-8). Even the latest Randomized Controlled Trial (RCT) (a large ongoing RCT, the American College of Surgeons Oncology Group (ACOSOG)-Z6051 trial) also excludes such advanced cases (11).

In this report, 11 patients who underwent L-ETME with multi-visceral resection were reviewed. Although this cohort was very small, L-ETME was found to have several advantages over open surgery. First, laparoscopic surgery provided high-definition, illuminated, and magnified images of 
the small structures in the narrow pelvis, which made it easier to identify important tissues including lymphatic tissues and autonomic nerve plexuses. The technological advances in instrumentation, advances in surgical techniques, and increased surgeon experience have made L-ETME more precise and easier to perform (10). In addition, creation of pneumoperitoneum may reduce oozing from small vessels and keep the operative field dry. Fujita et al. reported operation time of $375 \mathrm{~min}$ and mean blood loss of $1066 \mathrm{ml}$ in patients who underwent laparoscopic rectal cancer surgery with lateral pelvic node dissection (LPLD) (19). Moriya et al. reported mean operation time and blood loss of $393 \mathrm{~min}$ and $2128 \mathrm{ml}$, respectively, for 53 patients undergoing LPLD with internal iliac vessel excision (20). In a recent systematic review of open pelvic exenteration for advanced rectal cancer, the median complication rate was $57 \%$ (range $=37-100 \%$ ) (14). In the present series, mean operation time and blood loss were 365 min and $310 \mathrm{ml}$, respectively, which were both appropriate. In addition, the rate of postoperative complications was $27.3 \%$ (3/11), which was also less than rates previously reported. Only one patient had a postoperative complication of urinary dysfunction that needed urological medication in the present study. Unfortunately, multi-visceral resection has a risk of this type of complication, although no deaths and no re-operations occurred in the present series.

Second, the laparoscopic approach in ETME with multivisceral resection reduces the risk of peritoneal adhesions. Patients who need ETME with multi-visceral resection have a high probability of locoregional recurrence and may require repeated operations. In such cases with repeated operations, laparoscopic surgery may provide an easier surgical approach for colorectal surgeons (4-6).

The multi-center, randomized, controlled trial CLASICC provided evidence of equivalent 3-year and 5-year local recurrence, disease-free survival, and overall survival rates of laparoscopic surgery compared to open surgery (8). However, there was no subgroup analysis of locally advanced rectal cancer (cT4 tumors) in this trial, and the maturity of the technique of laparoscopy has been questioned due to a relatively high conversion rate and morbidity. The 3-year OS and 3-year LRFS rates in the present series were good and comparable to other reported results (8-11). This suggests that the magnified view of laparoscopy can accurately capture the changes in the surrounding tissue due to the tumor, and it enables selection of the appropriate dissection layer better than the sense of touch in open surgery.

There were several limitations in the present study. First, a very small number of patients was enrolled. Second, this study was retrospectively conducted at a single institution. Third, short-term results were reported, because the median follow-up time was less than 5 years. Further studies with a long-term follow-up are needed to verify the feasibility and efficacy of L-ETME for locally advanced lower rectal cancer.
Despite the small number of patients enrolled in the present study, the results demonstrate that L-ETME is a feasible and safe procedure for locally advanced rectal cancer.

\section{Conclusion}

The results of the present study suggest that L-ETME is feasible and has efficacy for locally advanced lower rectal cancer.

\section{Conflicts of Interest}

No funding was received for the present study and there are not conflicts of interest to declare.

\section{References}

1 Jacobs M, Verdeja JC and Goldstein HS: Minimally invasive colon resection (laparoscopic colectomy). Surg Laparosc Endosc 1: 144-150, 1991.

2 Buchanan GN, Malik A, Parvaiz A, Sheffield JP and Kennedy RH: Laparoscopic resection for colorectal cancer. Br J Surg 95: 893-902, 2008.

3 Neudecker J, Klein F, Bittner R, Carus T, Stroux A and Schwenk W: Short-term outcomes from a prospective randomized trial comparing laparoscopic and open surgery for colorectal cancer. Br J Surg 96: 1458-1467, 2009.

4 Park JS, Choi GS, Lim KH, Jang YS, Kim HJ, Park SY and Jun SH: Laparoscopic extended lateral pelvic node dissection following total mesorectal excision for advanced rectal cancer: initial clinical experience. Surg Endosc 25: 3322-3329, 2011.

5 Ishizaki H, Nakashima S, Hamada T, Nishida T, Maehara N, Ikeda $\mathrm{T}$, Tsukino $\mathrm{H}$, Mukai $\mathrm{S}$, Kamoto $\mathrm{T}$ and Kondo $\mathrm{K}$ : Laparoscopic anterior pelvic exenteration for locoregionally advanced rectal cancer directly invading the urinary bladder: A case of low anterior resection with en bloc cystectomy for sphincter preservation. Asian J Endosc Surg 8: 343-346, 2015.

6 Zhou ZX, Zhao LY, Lin T, Liu H, Deng HJ, Zhu HL, Yan J and Li GX: Long-term oncologic outcomes of laparoscopic $v s$. open surgery for stages II and II rectal cancer: A retro-spective cohort study. World J Gastroenterol 21: 5505-5512, 2015.

7 Akiyoshi T: Technical feasibility of laparoscopic extended surgery bryond total mesorectal excision for primary or recurrent rectal cancer. World J Gastroenterol 22: 718-726, 2016.

8 Jayne DG, Thorpe HC, Copeland J, Quirke P, Brown JM and Guillou PJ: Five-year follow-up of the Medical Research Council CLASSIC trial of laparoscopically assisted versus open surgery for colorectal cancer. Br J Surg 97: 1638-1645, 2010.

9 Kang SB, Park JW, Jeong SY, Nam BH, Choi HS, Kim DW, Lim SB, Lee TG, Kim DY, Kim JS, Chang HJ, Lee HS, Kim SY, Jung KH, Hong YS, Kim JH, Sohn DK, Kim DH and Oh JH: Open versus laparoscopic surgery for mid or low rectal cancer after neoadjuvant chemotherapy (COREAN trial): short-term outcomes of an open-label randomized controlled trial. Lancet Oncol 11: 637-645, 2010.

10 van der Pas MH, Haglind E, Cuesta ME, Fürst A, Lacy AM, Hop WC and Bonjer HJ: Laparoscopic versus open surgery for rectal cancer (COLOR II): short-term outcomes of a randomized, phase 3 trial. Lancet Oncol 14: 210-218, 2014. 
11 Nandakumar G and Fleshman JW: Laparoscopy for rectal cancer. Surg Oncol Clin Nam 19: 793-802, 2010.

12 Ishiguro S, Akasu T, Fujita S, Yamamoto S, Kusters $M$ and Moriya Y: Pelvic exenteration for clinical T4 rectal cancer: oncologic outcome in 93 patients at a single institution over a 30-year period. Surgery 145: 189-195, 2009.

13 Mukai T, Akiyoshi T, Ueno M, Fukunaga Y, Nagayama S, Fujimoto Y, Konishi T, Ikeda A and Yamaguchi T: Laparoscopic total pelvic exenteration with en bloc lateral lymphnode dissection after neoadjuvant chemotherapy for advanced primary rectal cancer. Asian J Endosc Surg 6: 314-317, 2013.

14 Nagasaki T, Akiyoshi T, Ueno M, Fukunaga Y, Nagayama S, Fujimoto $\mathrm{Y}$, Konishi $\mathrm{T}$ and Yamaguchi $\mathrm{T}$ : Laparoscopic abdominosacral resection for locally advanced primary rectal cancer after treatment with mFOLFOX6 plus bevacizumab, followed by preoperative chemoradiotherapy. Asian J Endosc Surg 7: 52-55, 2014.

15 Kim KY, Hwang DW, Park YK and Lee HS: A single surgeon's experience with 54 consecutive cases of multivisceral resection for locally advanced primary colorectal cancer: Can the laparoscopic approach be performed safely? Surg Endosc 26: 493-500, 2012.

16 Dindo D, Demartines $\mathrm{N}$ and Clavien PA: Classification of surgical complications: a new proposal with evaluation in a cohort of 6336 patients and results of a survey. Ann Surg 240: 205-213, 2004
17 Hermanek $\mathrm{P}$ and Junginger T: The circumferential resection margin in rectal carcinoma surgery. Tech Coloproctol 9: 193200, 2005.

18 Larsen SG, Wiig JN, Emblemsvaag HL, Grøholt KK, Hole KH, Bentsen A, Dueland S, Vetrhus T and Giercksky KE: Extended total mesorectal excision in locally advanced rectal cancer (T4a) and the clinical role of MRI-evaluated neo-adjuvant downstaging. Colorectal Dis 11: 759-767, 2009.

19 Fujita S, Yamamoto S, Akasu T and Moriya Y: Lateral pelvic lymphnode dissection for advanced lower rectal cancer. Br J Surg 90: 1580-1585, 2003.

20 Moriya Y, Hojo K, Sawada T and Koyama Y: Significance of lateral node dissection for advanced rectal carcinoma at or below the peritoneal reflection. Dis Colon Rectum 32: 307-315, 1989.
Received February 17, 2018

Revised March 6, 2018

Accepted March 7, 2018 